\title{
Pneumatic Muscle Actuated Compliant Gripper Systems
}

\author{
Andrea Deaconescu \\ Transilvania University of Brasov, Industrial Engineering and Management Department, Faculty of Technological Engineering and Industrial \\ Management, Romania
}

\begin{abstract}
The paper presents the stages of developing new, light, eco-friendly and bionic gripper systems. Gripping is achieved by means of original, self adaptive, bio-inspired systems, with a pneumatic muscle as motion generator. The method underlying the development of these new gripping systems is based on the creation of concepts by analogy, an instrument aimed at widening the inspiration horizon in designing by using models from nature.
\end{abstract}

\section{Introduction}

Amongst the numerous functions of the human hand the most important one is grippping. While the grasping capacity of the extremities of the limbs can also be found in animals (e.g. lobster claws), it is in humans that it has reached its highest functionality. Thus, various combinations of the independent motions of the finger segments (phalanges) of the human hand allow gripping, holding, moving and releasing of various bodies.

The gripping function in humans entails a sequence of phases, starting with choosing the grasping method, followed by the actual seizing action and ending with controlling the manipulated object. Gripping is a consequence of desire generated internally or externally, that causes reactions at visual and brain level. Hence the hand is positioned close to the envisaged object, seizes it and carries out the programmed task. Figure 1 shows the phases entailed by gripping and the links between these $[1,2]$.

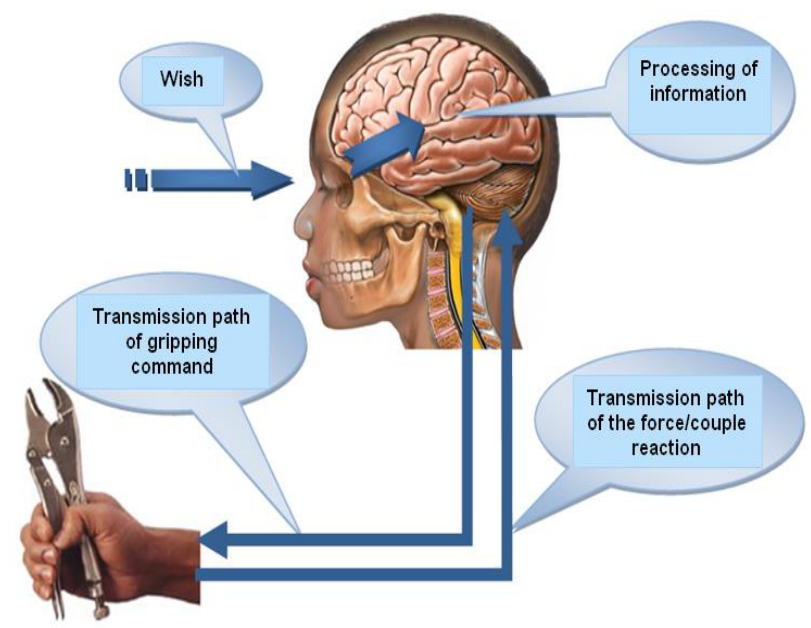

Figure 1. Command flows of a human hand gripping action.
In the field of robotics gripping entails the contact between the effector element of a robot (the gripper) and a body in view of its manipulation. The grippers are those components of robot systems that facilitate the temporary contact with the manipulated object, ensuring its position and orientation during transport and assembly.

The main functions of gripping systems depend on the specific applications they are part of and include [3]:

- temporary maintaining of a final position and orientation of a body in relation to the manipulation equipment;

- firm holding of the gripped object under static conditions (und the action of its own weight), dynamic conditions (in motion, during acceleration and braking) or when subjected to the action of external forces (cutting forces, torques);

- determining and modifying the position and orientation of the object depending on the concrete requirements of the application, etc.

In order to carry out the above functions, a gripping system needs to include the following main components:

- an energy system, meant to provide the type and quantity of energy required by the gripper;

- a mechanical execution system that carries out the actual gripping task;

- a system of sensors and transducers, meant to collect information (forces, displacements, accelerations etc.) and to transmit these to the control system;

- an information processing and control system that receives the signals coming from the sensors and transducers, processes these and consequently issues the necessary commands to the energy system.

The studies discussed in this paper are meant to present novel, light and eco-friendly gripper systems of bionic type. Gripping is achieved by means of original, self adaptive, bio-inspired systems, with a pneumatic muscle as motion generator. The proposed solutions of gripper systems are of non-anthropomorphic type 
designed for industrial or medical robotics applications.

The method underlying the development of these new gripping systems is based on design by analogy, and is a powerful working instrument aimed at widening the inspiration horizon in designing, at increasing the number of proposed variants, and consequently at generating a data base of solutions for gripper systems usable in various applications.

\section{Design by analogy. Biomimetics}

Design by analogy represents a frequently utilized method for the generation of new products and/or technologies. Numerous scientific papers address the issue of systematic knowledge transfer from natural sciences, from biology to engineering, and argue the enormous potential of bionic design (biomimetics) for developing new products and technologies [4].

The functional morphology of living organisms regarded from an engineering perspective represents a permanent source of inspiration for innovative solutions of high-tech constructions. In this respect since 1960 bionics (biomimetics), a novel scientific branch has developed, combining knowledge from biology, mathematics, medicine and engineering. Bionics draws upon biological intuition and engineering pragmatism to adapt nature's projects to the requirements of modern technics. Nature is but the starting point for innovations, it only offers clues as to what is useful in a mechanism. Starting from such clues it is the task of engineers to develop and refine the analyzed system. Thus bionics represents systematic learning from nature, as opposed to "inspiration from (copying) nature" $[5,6]$.

The motive for that bionics has not yet reached maturity from an engineering point of view, is that nature is an unbounded and impossible to control complex. Nature does not "design" a structure to attain a final target, as would an engineer; it encompasses billions of random experiments conducted over thousands of generations yielding beings whose aim it is to survive long enough such as to produce the next generation and launch the next round of random experiments.

Globally bionics is increasingly active, some of its achievements being, for example, the studies completed in England and the USA concerning the protuberances of the front side of the humpback whale tail with applications in the geometry of the airplane wing, and the studies conducted in Germany on the finger-shaped feathers of raptor birds that inspire engineers in creating airplane wings with geometries variable during flying, such as to reduce air resistance and fuel consumption. Medical research in Japan was aimed at reducing the pain caused by an injection by means of serrated edge hypodermic needles similar to a mosquito's proboscis, such as to minimise nerve stimulation.

Among the objectives of current bionic research, special attention comes to the study of effector organs. Their study, as well as that of the transmission processes of commands to them represents an essential part of bionics. In this respect terrestrial animals, birds and fish represent the source of inspiration for the development of various motion generating systems. Such an example is the study of the lizard's climbing motion and the subsequent transfer of the obtained results to the project of a climbing robot (Fig. 2a). Another example is that of an aeroplane wing, a constructive solution inspired from the wing of a bat (Fig. 2b).
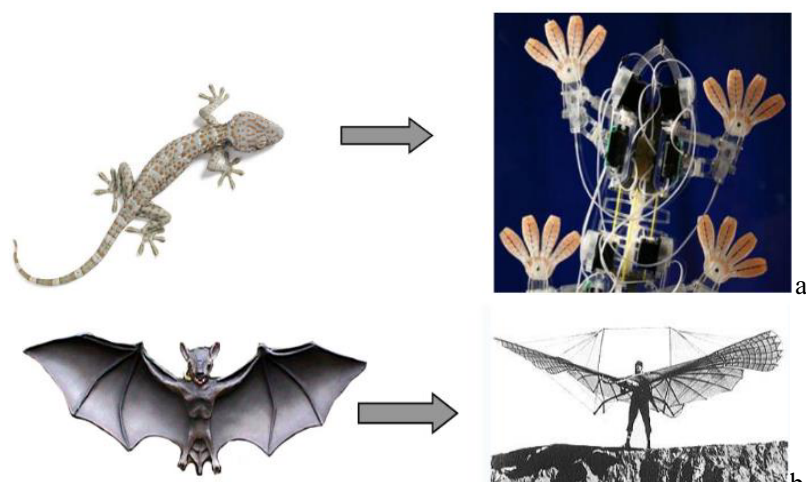

Figure 2. Examples of biomimetics.

\section{Gripper systems with bionic actuation}

An essential problem related to the design of a novel gripper system concerns the functional characteristics this has to fulfil. Requirements like the generated force, rigidity/compliance, dexterity, the number of degrees of mobility depend on the application the gripper is designed for.

Natural gripper systems represent an inexhaustible source of inspiration for engineers, and in time they have underlain numerous practical applications. Thus figure 3 presents a mechatronic system bio-inspired from the seizing of an egg by a person using two fingers and based on this the accordingly developed bio-inspired mechatronic system [2].

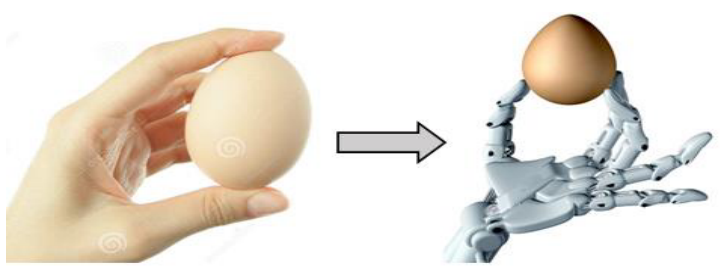

Figure 3. Transfer of the natural gripper model to an artificial system.

An artificial gripper system needs to ensure not only the seizing of the object, but also its safe manipulation. Thus, in the case of the egg of the above example, the magnitude of the applied force needs to be controlled, such as to not deteriorate the seized object. This is the case on compliant behaviour (Figure 4).

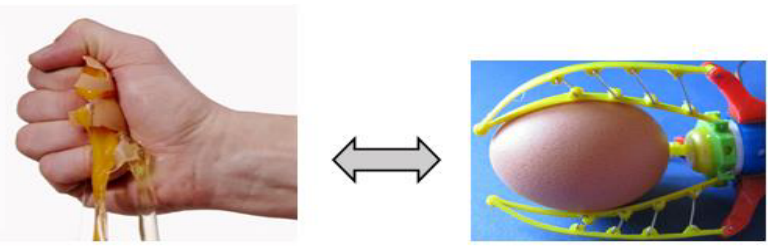

Figure 4. Seizing the gripped object with an uncontrolled vs. controlled force. 
Compliance, the inverse of rigidity, represents one of the most important properties required from a gripper system. A compliant gripper system allows the safe manipulation of objects, without causing their deformation, by applying of forces of adaptable magnitude. Such behaviour can be ensured by deploying adjustable compliant actuators - ACAs, like pneumatic muscles.

Figure 5 presents some of the main phases of the gripping process, namely approaching the object to be seized, initiation of the contact and its firm gripping between the two jaws by the application of an increasing force. For these phases the classic profile of the jaws' velocities is presents, as well as the desired and necessary form of the curve that describes the compliance of the entire system.

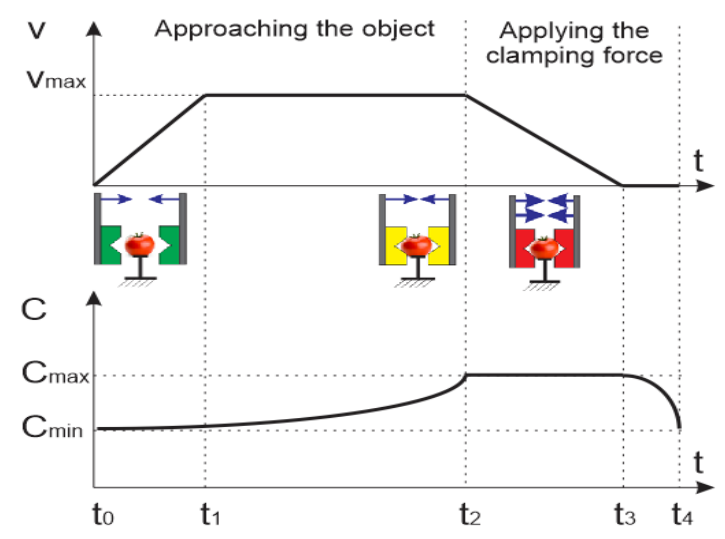

Figure 5. Variation of the jaw velocity and of the compliance of a gripper versus time.

The two graphs suggest that in the absence of contact between the jaws and the object, between moments $t_{0}$ and $t_{2}$ compliance can have smaller values, what allows a better positioning precision given by higher rigidity. The form of the curve that describes the variation of compliance needs to be concave, what allows a greater increase of compliance towards the moment of jaw-object contact initiation.

Between moments $t_{2}$ and $t_{3}$, when contact has been achieved, compliance needs to have the greatest values, thus ensuring the safe gripping of the object without its deformation/destruction.

An adjustable compliant actuator, like the pneumatic muscle, can adapt its functional behaviour between two limits. Thus its behaviour can vary from very rigid required for a good positioning precision - to compliant, when the main requirement is the safety of the motion.

From the above follows that novel, innovative, bioinspired solutions of gripper systems require light, flexibly structured equipment, with a high useful load-toeigen-weight ratio, with integrated position adjustment and force control at an affordable price. Currently no gripper systems are available that would satisfy the majority of these requirements.

The paper aims at presenting innovative bio-inspired gripper systems the novelty of which consists in their actuation, namely with pneumatic muscles. In this respect the principles of design by analogy are applied via a topdown bionic approach. Such approach entails that subsequently to stating the problem, natural systems are to be identified able to provide ideas for adequate solutions.

Gripping is achieved by means of mechanical contact forces. While in natural systems these forces are generated by a muscle, in artificial grippers forces are generated by motors. In the novel gripper systems proposed in this paper the deployed motor is a pneumatic muscle, a bio-inspired system analogous to human and animal muscles.

Between the motor and the jaws (the effectors) typically a mechanism intervenes, consisting of rigid elements. In this case the proposed gripper systems are based on gears.

The pneumatic muscle copies by biomimetics the functioning of the human muscle fibre (Figure 6), and has a number of characteristics like shock absorbing capacity and shock resistance, low weight, small overall dimensions and mass per unit of power and elasticity [7]. These characteristics render pneumatic muscles optimum constructive elements for applications in robotics, for both orientation and gripper mechanisms.

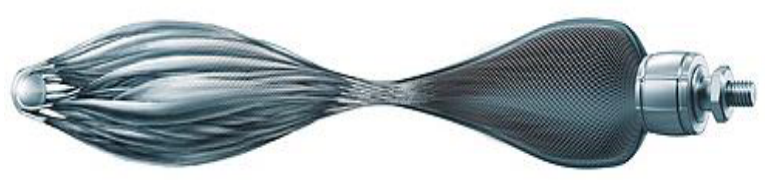

Transition from the human to the pneumatic muscle

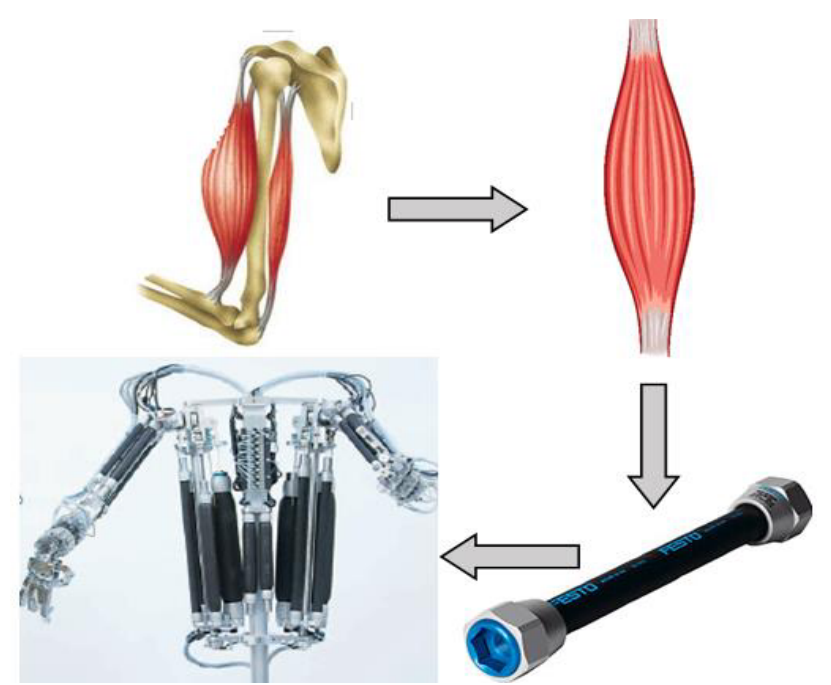

Figure 6. Pneumatic artificial muscles mimic a biological muscle.

Further on three variants of a gripper system are proposed, with a pneumatic muscle as the motor element linked to a gear mechanism for power transmission.

The pneumatic muscle actuated gripper systems were dimensioned based on the following input data: mass of the object $\mathrm{m}=0.7 \mathrm{~kg}$; acceleration of the motion of the system consisting of the gripper and the object: $a=5 \mathrm{~m} / \mathrm{s}^{2}$; gravitational acceleration: $g=9.81 \mathrm{~m} / \mathrm{s}^{2}$; emergency stop deceleration: $\mathrm{a}_{\mathrm{S}}=10 \mathrm{~m} / \mathrm{s}^{2}$; friction coefficient: $\mu=0.2$; safety coefficient: $S=2.5$. Considering the imposed requirements related to functioning and overall dimensions, the models proposed in this paper are based on the utilization of the smallest pneumatic muscle 
produced by Festo, Germany, (MAS-10-45N-AA-MC-OER-EG), with the following characteristics:

- muscle diameter: $10 \mathrm{~mm}$;

- length of the active part: $45 \mathrm{~mm}$;

- maximum working pressure: 6 bar.

The maximum forces that have to be developed by this pneumatic muscle occurr for the highest values of the accelerations, namely in sudden braking. In such a case the necessary gripping force the be exerted by one jaw is computed by equation (1):

$$
F_{G}=\frac{m \cdot\left(g+a_{S}\right) \cdot S}{\mu \cdot n}=\frac{0.7 \cdot(9.81+10) \cdot 2.5}{0.2 \cdot 2}=86.67 \mathrm{~N}
$$

The proposed gripper systems have two jaws, thus requiring the selected pneumatic muscle to develop a force of minimum $2 \cdot 86.67 \mathrm{~N}=173.34 \mathrm{~N}$.

Figure 7 presents the graph describing the force developed by the selected pneumatic muscle versus pressure and stroke. The hatched rectangle marks the optimum deployment area of the pneumatic muscle, so that for a $4 \mathrm{~mm}$ maximum stroke of the jaws the developed force exceeds $173.34 \mathrm{~N}$.

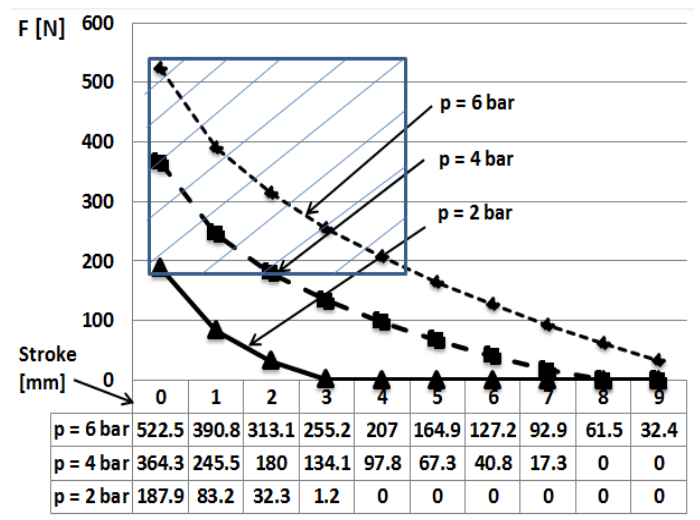

Figure 7. Variation of the developed force versus pressure and stroke.

Figures 8 to 10 show the schematics of the three variants of gripper systems. All the three proposed variants of gripper systems benefit from compliant behaviour, thus ensuring the safe manipulation of fragile objects, without deforming or destroying them. The utilization of a pneumatic muscle as an adjustable compliant actuator allows deviations from the position of equilibrium, their magnitude depending on the level of straining external forces.

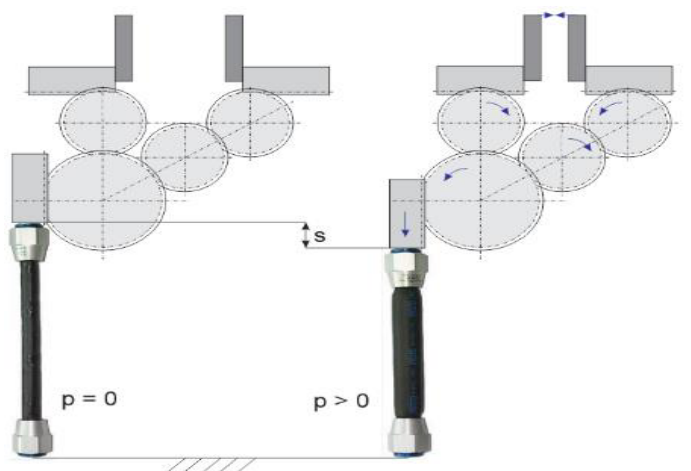

Figure 8. Variant 1 (V1): Parallel, asymmetrical gripper system with two mobile jaws.

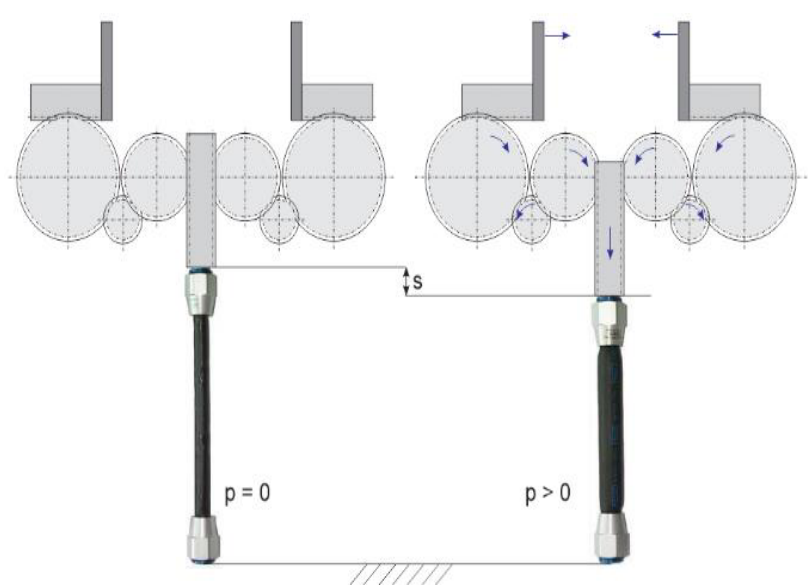

Figure 9. Variant 2 (V2): Parallel, symmetrical gripper system with two mobile jaws.

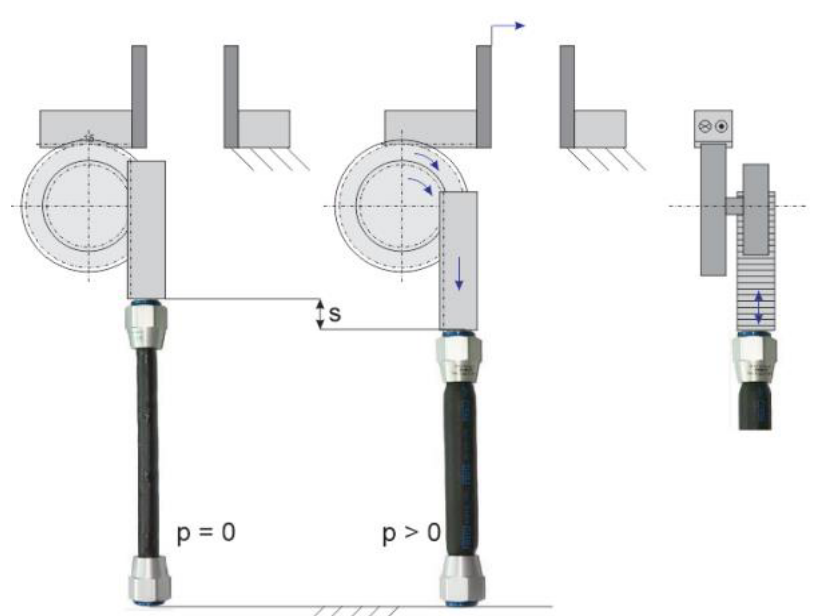

Figure 10. Variant 3 (V3): Parallel, asymmetrical gripper system with one mobile and one fixed jaw.

From the three proposed variants the asymmetrical one with two mobile jaws was built as a prototype and tested. Figure 11 shows the experimental model.

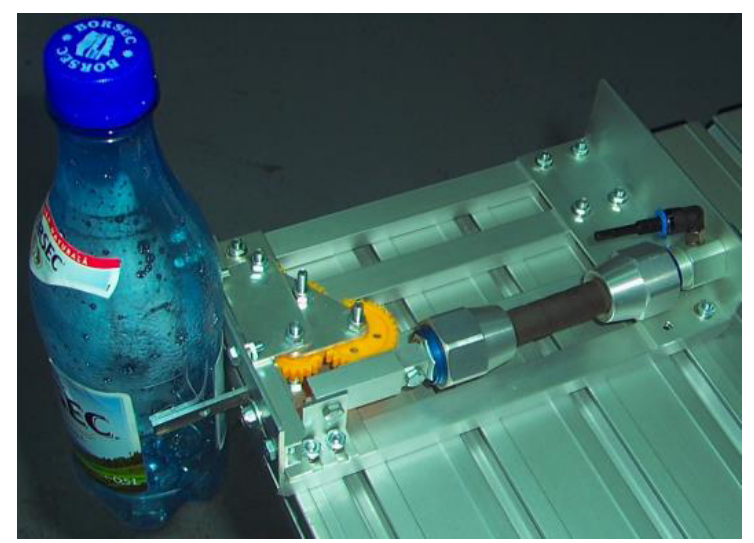

Figure 11. Parallel, asymmetrical gripper system with two mobile jaws.

The power transmission mechanism consists of gears and racks that ensure the final motion of the two gripping jaws. The pneumatic muscle is positioned asymmetrically, thus allowing the metal structure of the gripper system to include the proportional pressure regulator that controls the motion of the linear motor. The displacement of the jaws is achieved by means of an MPPES-3-1/4-6-010 
proportional pressure regulator (manufactured by Festo) that can be controlled by a MPZ-1-24DC-SGH-6-SW reference module. This actuation concept allows the slow and uniform charging of the pneumatic muscle, while avoiding possible shocks being introduce into the system.

Of particular interest within the conducted experiments was determining the compliant and adjustable character of the gripper system. Adjustable compliance is obtained only for a non-linear dependency of the force developed by one jaw on its displacement. An adjustable compliant actuator, as is the pneumatic muscle, can adapt its behaviour between two limits. Thus its behaviour can vary from very rigid (required for a good positioning accuracy) to compliant, when the main requirement is ensuring motion saftey.

Figure 12 presents the variation of the force developed by one jaw versus its stroke, the pressure varying from 0 to 6 bar. It can be observed that at the beginning of the motion the value of the force is at its maximum, and as the jaw progresses towards its limit, the force decreases.

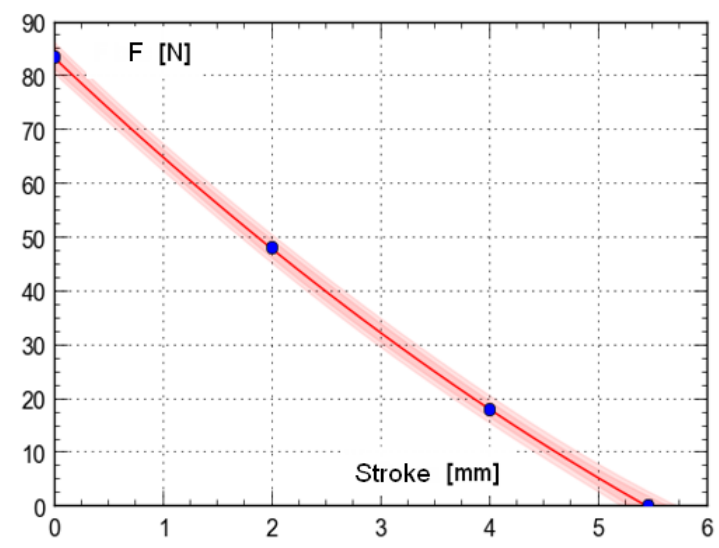

Figure 12. Variation of the force developed by one jaw vs its stroke

The regression function corresponding to the previous curve is (2):

$$
F_{j a w}=83.36-19.146 \cdot c+0.7088 \cdot c^{2}
$$

The compliance $C$ of the gripper system is computed as the inverse of rigidity $(k)$ :

$$
C=k^{-1}=\left(-\frac{d F}{d c}\right)^{-1}=\frac{1}{19.146-1.4176 \cdot c}
$$

Figure 13 shows the evolution of compliance versus the stroke of a jaw.

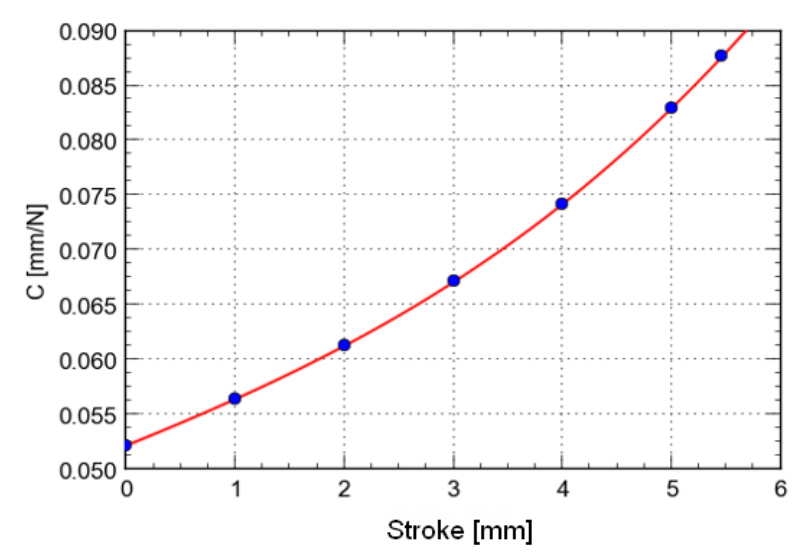

Figure 13. Compliance of the gripper system.

The above graph reveals the concave shape of the compliance curve, what facilitates the accelerated increase of this quantity once the jaw-object contact has been achieved.

\section{Conclusions}

The paper has presented several innovative constructive solutions of non-anthropomorphic gripper systems that ensure the safe seizing and manipulation of fragile objects. The idea of utilizing pneumatic muscles as actuators was taken from nature, by biomimetics, based on the functioning of the human muscle fibre.

The utilization of the linear pneumatic muscle as the actuator of the gripper system entails a light construction of significantly compliant behaviour, which corresponds to the safety requirements concerning the interaction with humans.

\section{References}

1. S. Nof (editor), Handbook of Industrial Robotics. (John Wiley and Sons Inc. New York, (1985)

2. D. Negrea - Research concerning the gripper systems of industrial robots actuated by pneumatic muscles. (PhD Thesis Universitatea Transilvania din Braşov, 2014)

3. G.J. Monkman, S. Hesse, R. Steinmann, H. Schunk, Robot Grippers. (WILEY-VCH Verlag GmbH \& Co. KGaA, Weinheim, 2007)

4. B.T. Christensen, C.D. Schunn, The Relationship of Analogical Distance to Analogical Function and Preinventive Structure: The Case of Engineering Design. Memory \& Cognition, 35(1) (2007)

5. G.E. Dieter, Engineering Design: A Material and processing Approach. (McGraw-Hill, 2000)

6. K.T. Ulrich, S.D. Eppinger, Product Design and Development. (Boston McGraw-Hill/Irwin, 2004)

7. Festo. Precisely to the point: membrane technology. https://www.festo.com/cms/en_corp/14047.htm Accessed 2015 